\title{
Pulmonary haemosiderosis (apparently idiopathic) associated with myocarditis, with bilateral penetrating corneal ulceration, and with diabetes mellitus
}

\author{
K . J . M U R P H Y \\ From the Princess Alexandra Hospital, Ipswich Road, Woolloongabba, S.2, Queensland, Australia
}

The literature of pulmonary haemosiderosis includes an account by Campbell and Macafee (1959) of idiopathic pulmonary haemosiderosis associated with myocarditis. Their patient first had symptoms of shortness of breath at the age of 7 ; he was found to have the radiological changes of pulmonary haemosiderosis at the age of 12 , and he died when 14 years old. Death was due to cardiac failure, and at necropsy the heart showed a marked degree of fibrosis and there was infiltration by polymorphs, mononuclears, and lymphocytes.

The following case is reported because of the appearance of pulmonary haemosiderosis and the subsequent development of cardiac manifestations plus a number of other features.

\section{CASE REPORT}

The patient was a meatworker who first developed a penetrating corneal ulcer in the left eye when 19 years old. He was admitted to Townsville Hospital for excision of a prolapsed iris and was found to have diabetes mellitus. At the age of 21 he developed a perforating corneal ulcer of the right eye, and again required excision of the prolapsed iris. This eye continued to be irritable for the next 12 months, and when corneal perforation again seemed to be imminent he was readmitted. A routine chest radiograph (Figs 1a and 1b) showed fine miliary nodulation in the lower zones of both lungs: the cardiac outline was normal. There were no cardiac or respiratory symptoms at this stage. He was readmitted because of the eye condition six months later, and was still free of cardiac and respiratory symptoms.

When 24 years old he was admitted because of severe breathlessness and haemoptysis for three weeks, during which he had been easily fatigued and had noted some irregularity of his heart beat. On admission the pulse was regular, the blood-pressure was $140 / 70 \mathrm{~mm} . \mathrm{Hg}$, and there was a triple rhythm but no murmur. A chest radiograph at this time (Fig. 2) showed that the fine nodulation of the lower lung fields was more obvious and there was some increase in the cardiac diameter. The patient had an irregular fever to $100.6^{\circ} \mathrm{F}$, which subsided spontaneously after three weeks. The haemoglobin was 8.1 g. $/ 100 \mathrm{ml}$., and a blood smear showed marked hypochromia and microcytosis. The white cell count was $8,500 / \mathrm{c} . \mathrm{mm}$. with a normal differential. E.S.R. was $100 \mathrm{~mm} . / \mathrm{hr}$ (Westergen). Serum bilirubin was $0.3 \mathrm{mg} . / 100 \mathrm{ml}$; there was no albuminuria, and microscopic examination of the urine showed no abnormality. Sputum culture grew $\alpha$-haemolytic streptococci, and subsequent cultures for tubercle bacilli were negative. An occult blood test on the faeces was negative. Serum protein was $8 \mathrm{~g} . / 100 \mathrm{ml}$. with a normal electrophoretic pattern. Barium meal was normal. No L.E. cells were found in the peripheral blood.

The haemoptysis ceased, and with intramuscular iron therapy the haemoglobin rose to $14.7 \mathrm{~g} . / 100 \mathrm{ml}$. The E.S.R. fell to $28 \mathrm{~mm}$./ $\mathrm{hr}$ and the blood smear returned to normal. There was a transient eosinophilia of up to $11 \%$ of a total count of 14,500 white cells. Original electrocardiograms showed a right bundle branch block. The right bundle branch block became intermittent and finally disappeared, but later tracings showed frequent episodes of 2 to 1 heart block. There was no evidence of pulmonary oedema or of fluid retention, and there was no need for either digitalis or diuretics. The patient was discharged after two months, and at the time of discharge he was asymptomatic with a normal haemoglobin but with no alteration in the chest radiograph.

During the next six months the patient had a number of episodes of complete heart block and periods of Stokes-Adams syncope, and because of this he was referred to Brisbane for further investigation. He was then aged 25 years. There was no finger clubbing or cyanosis: the blood-pressure was $120 /$ 


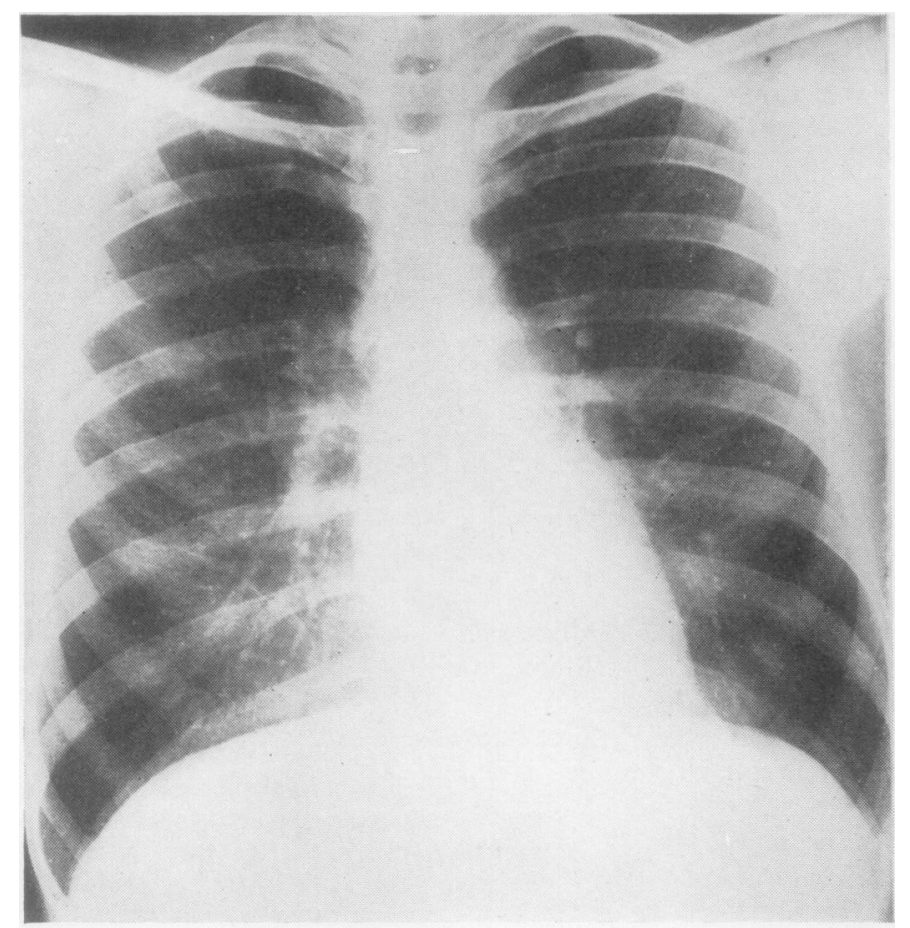

(a)

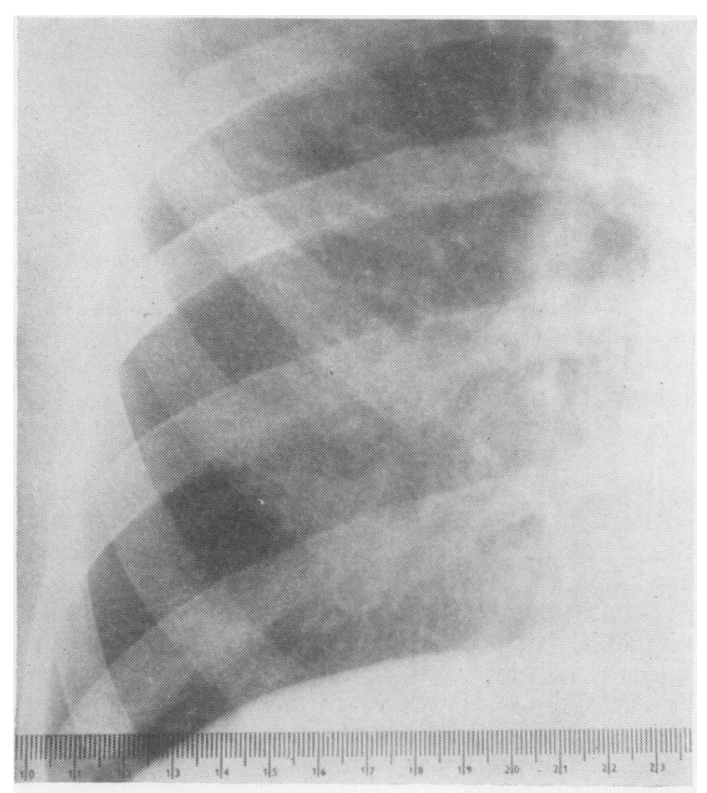

(b)

FIG. 1. a, Routine chest radiograph at age 22. Fine nodulation is present in both lower zones. Cardiac silhouette normal. b, Right lower lung field from Fig. $1 a$.
$80 \mathrm{~mm} . \mathrm{Hg}$, and there was no heart murmur. Ankle jerks were absent but other reflexes were normal, and sensation was normal. There were firm, discrete, non-tender lymph nodes up to $2 \mathrm{~cm}$. in diameter in both axillae; groin glands were less than $1 \mathrm{~cm}$. An electrocardiogram showed complete heart block with left bundle branch block. The atrial rate was 100 per minute and the ventricular rate 40 per minute. He still had bilateral corneal ulcers with loss of aqueous from both eyes, but the corneal sensation was normal, and as far as could be seen the retinal vessels were normal. Swabs of the nose and throat were negative for $C$. diphtheriae, but since $\beta$-haemolytic streptococci and Streptococcus viridans were grown from the throat, intramuscular penicillin ( 2 million units per day) was given. The anti-streptolysin titre was 625 Todd units/ml., the haemoglobin and smear were normal, as were the bleeding and clotting times, and there were no L.E. cells in the peripheral blood. Skin biopsy was normal, with no excess of iron. Biopsy of an axillary lymph node showed gross reactive changes with prominent germinal centre proliferation. He had a temporary return to sinus rhythm when he was given $90 \mathrm{mg}$. oral ephedrine per day. Complete heart block soon returned and was associated with episodes of ventricular standstill and Stokes-Adams syncope. In addition, the leaking corneal ulcers caused considerable concern.

Neither the patient nor his family could recall any history of diphtheria or of rheumatic fever, and so 


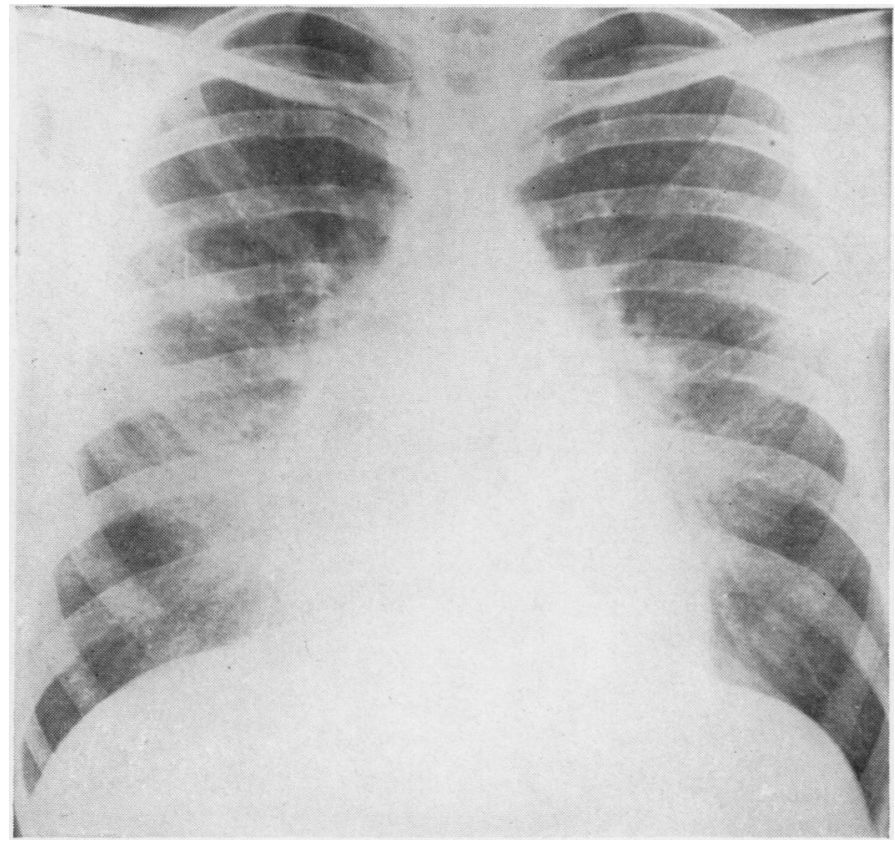

FIG. 2. Chest radiograph at age 24 at onset of symptoms. Lung nodulation is denser and more extensive, and heart is larger.

an operative lung biopsy was performed with a view to definitive diagnosis and as a preliminary to systemic steroid therapy. The right lung was found to be large and bulky, and to show generalized, quite marked fine emphysema of all lobes. On microscopic examination the lung showed many haemosiderinladen macrophages in the alveoli. There was emphysema, there was some hyperplasia of alveolar epithelial cells, and the walls of the alveoli were thickened (Fig. 3). There were chronic inflammatory cells in the alveolar walls, and there was some degree of intimal thickening of the blood vessels. Areas of more marked lung scarring showed haemosiderin impregnation of elastic tissue (Fig. 4). There was no evidence of pulmonary polyarteritis. Intercostal muscle taken at the same time was normal.

Oral prednisone was started in a dose of $60 \mathrm{mg}$. per day. Insulin requirements increased temporarily from 60 units per day to 80 units per day, but subsequently fell to 40 units per day. Two weeks after the prednisone was started the ulcers ceased to leak aqueous, and the anterior chambers of both eyes began to re-form. One month after the prednisone was commenced there was again a return to sinus rhythm, but this lasted for two weeks only. Episodes of syncope then became much more frequent, with up to eight episodes per day. In addition to the ephedrine, the patient was given isoprenaline without effect. Prednisone was gradually reduced to $30 \mathrm{mg}$. per day, but the episodes became even more frequent.
They were controlled for periods of up to 48 hours by intravenous molar sodium lactate to $200 \mathrm{ml}$. An external pacemaker was placed beside the patient's bed, but he died in one of his episodes of asystole before the pacemaker could be attached.

\section{NECROPSY}

The right lung weighed 680 g., the left lung $620 \mathrm{~g}$.: both had a brown appearance, particularly in the lower and middle lobes, and were firmer than normal. Microscopic examination of the lungs showed the same appearances of ironcontaining macrophages filling the alveoli, emphysema, and thickening of the alveolar walls. Some of the small blood vessels showed iron encrustation of the walls, but again no evidence of polyarteritis. The heart weighed $370 \mathrm{~g}$.; the wall of the left ventricle was $12 \mathrm{~mm}$. thick, and the wall of the right ventricle $2 \mathrm{~mm}$. thick. There was fibrosis of the posterior wall of the heart involving most of the muscle coat and extending into the endocardium. The heart valves were normal and the coronary vessels were patent with no evidence of polyarteritis. Microscopic examination of the heart confirmed the extensive fibrosis (Fig. 5). In addition there was much cellular infiltrate, largely with eosinophils and polymorphs, in the subendo- 


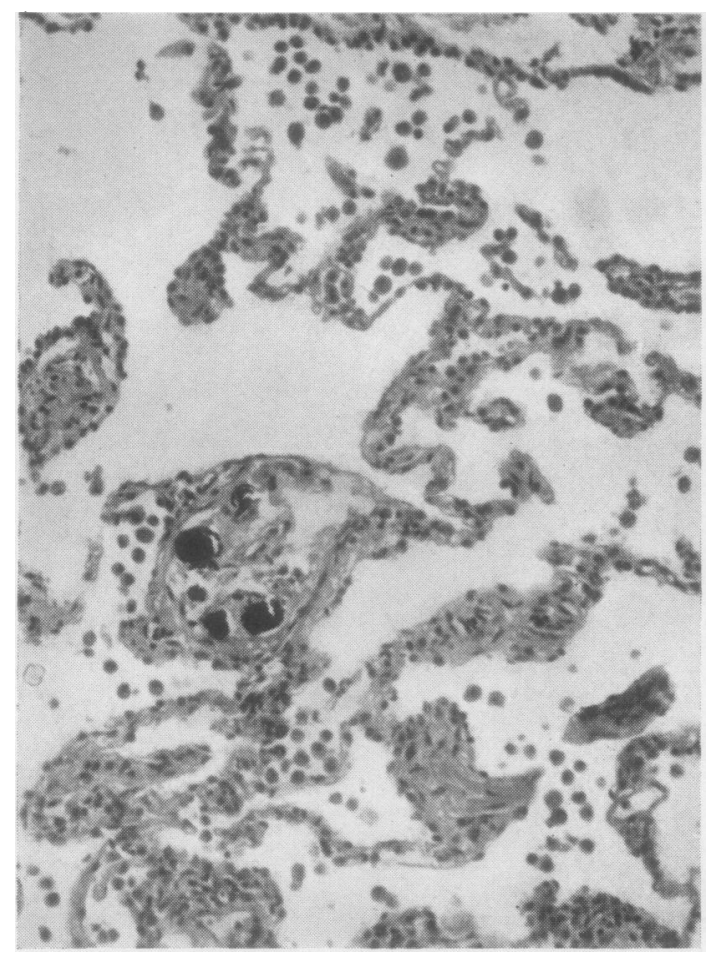

FIG. 3. Operative biopsy from surface of right lung. Alveolar walls are thickened and there is some hyperplasia of epithelial cells. Haemosiderin-laden macrophages are. present. Haematoxylin and eosin, $\times 50$.

cardial tissue of the left ventricle and in the left atrium (Fig. 6). The kidneys and the remainder of the body were normal. In particular, an extensive search for polyarteritis was negative. The left eye was sent to Dr. C. H. Greer, who reported that there was severe corneal ulceration and a mild diffuse uveitis but no evidence of polyarteritis. The right eye showed a similar appearance.

\section{DISCUSSION}

A diagnosis of idiopathic pulmonary haemosiderosis can be substantiated from the time of discovery of fine mottling on the chest radiograph even though the patient was asymptomatic at that stage. Wynn-Williams and Young (1956) pointed out that the original diagnostic feature may be only one of the triad of haemoptysis, anaemia, or radiological abnormality. Karlish (1962) reported a patient who had had neither haemoptysis nor anaemia at the time of diagnosis by the discovery of fine, granular mottling on the

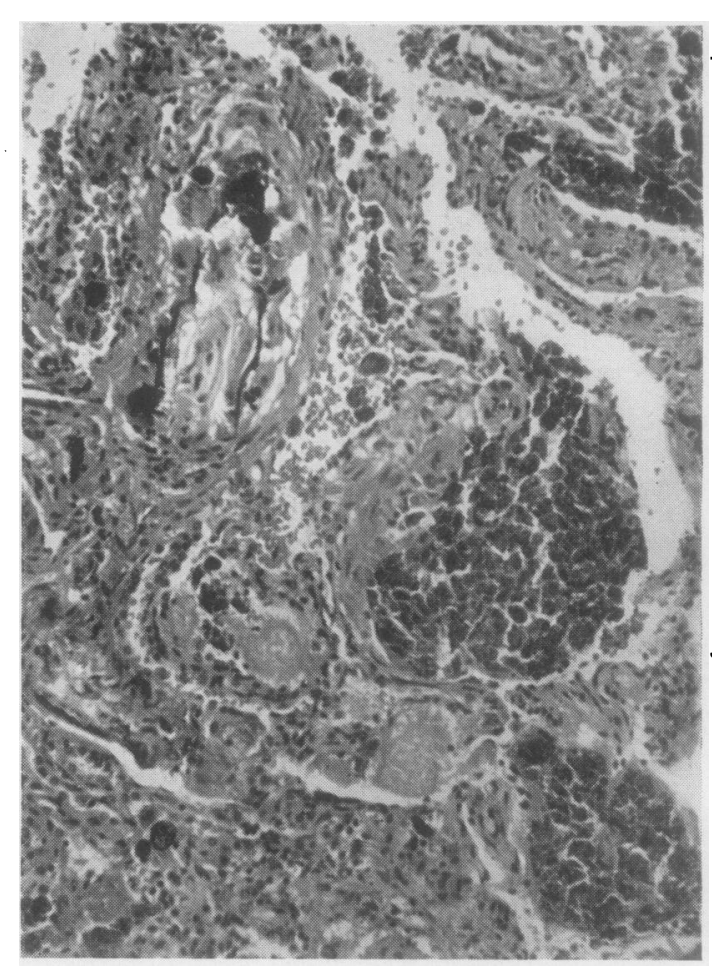

FIG. 4. Area of more dense pulmonary infiltration. There is iron impregnation of the elastic tissue of the vessel wall but no evidence of polyarteritis. Haematoxylin and eosin, $\times 50$.

chest radiograph and a confirmatory biopsy. When haemoptysis occurred two years later the lung pattern was more abnormal and the heart was $\delta$ larger.

Cardiac enlargement has been described in 웅 many cases accepted as idiopathic pulmonary haemosiderosis. Reye (1945) described a child who o died at the age of 4 with all cardiac chambers dilated but especially the right ventricle. He $\odot$ considered that the pulmonary haemosiderosis was $N$ idiopathic because there was no congestive cardiac N failure and no venous engorgement, and no $O$ cardiac enlargement when the lung lesions were first detected. Wyllie, Sheldon, Bodian and Barlow

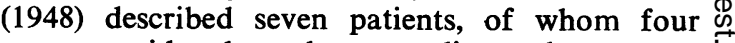
were considered to have cardiac enlargement: although these four were anaemic, so were the three others without cardiac enlargement. Necropsy on two of these showed histologically $\frac{\vec{\Phi}}{8}$ normal heart muscle. Walton and Williams (1951) noted slight cardiac enlargement in their patient when lung changes were first detected radio- o 


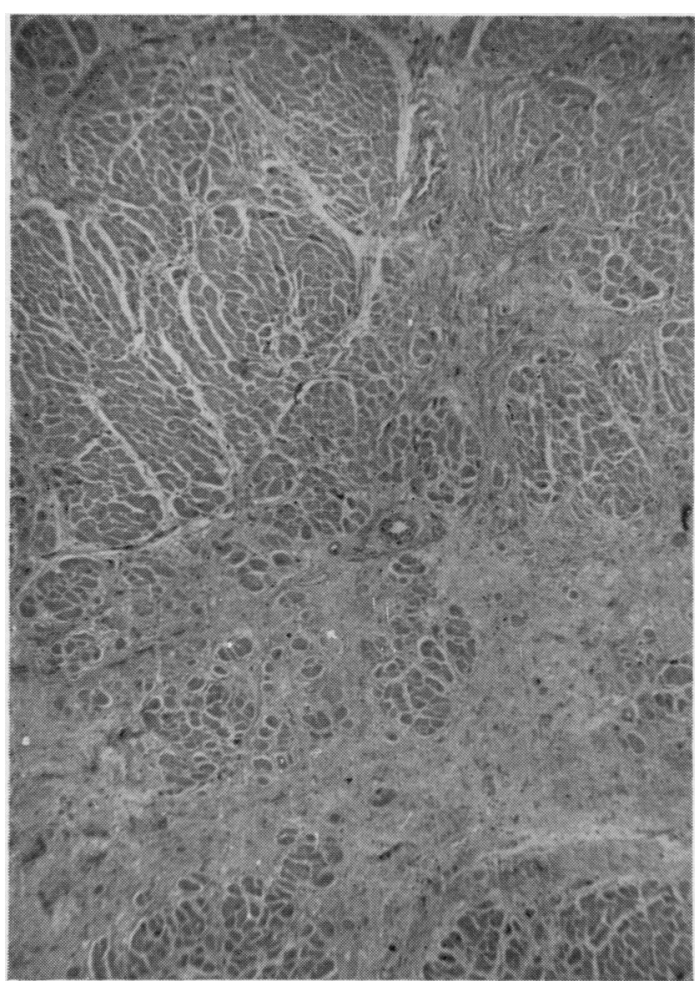

FIG. 5. Low-power view of left ventricle showing mature fibrous tissue. Haematoxylin and eosin, $\times 10$.

logically at the age of 17 years. Their patient died aged 19 of cardiac failure with a heart weight of $1,100 \mathrm{mg}$. at necropsy: no details of the histology of the heart were given. Campbell and Macafee (1959) found descriptions of five other cases in which myocardial change was mentioned. They considered that the pulmonary haemosiderosis in their own case was idiopathic and not secondary to myocarditis, mainly on cardiac catheter findings, which excluded pulmonary hypertension. Conversely, Wood (1954) considered that pulmonary haemosiderosis secondary to mitral stenosis did not have 'anything to do with pulmonary hypertension'.

Pulmonary haemosiderosis secondary to cardiac lesions, whether mitral stenosis or hypertensive left ventricular failure, was discussed by Lendrum, Scott, and Park (1950). They noted that the haemosiderosis was focal, with groups of four to 20 adjacent alveoli filled with haemosiderin-laden macrophages, and that these groups of affected alveoli were separated by normal alveoli. They considered that the histology in the affected areas was indistinguishable from that of idiopathic pulmonary haemosiderosis, and they found iron impregnation of elastic tissue of blood vessels in both conditions. They stressed the absence of emphysema macroscopically and microscopically in their cardiac cases.

The patient described in the present report had extensive macroscopic and microscopic emphysema. The haemosiderosis was diffuse although varying in degree in different areas. He also had an iron deficiency anaemia which responded to the administration of iron. When the heart disease became progressively worse the haemoptysis did not recur. The alveolar wall changes which Soergel and Sommers (1962) considered to be characteristic of idiopathic pulmonary haemosiderosis were present in both the biopsy and necropsy specimens.

There was no bronchiectasis. Cameron (1956) described cases of focal pulmonary haemosiderosis in bronchiectatic areas of patients with bronchiectasis, emphysema, and cor pulmonale.

There was no renal lesion to allow a diagnosis of Goodpasture's syndrome even though three years passed between the first radiological signs

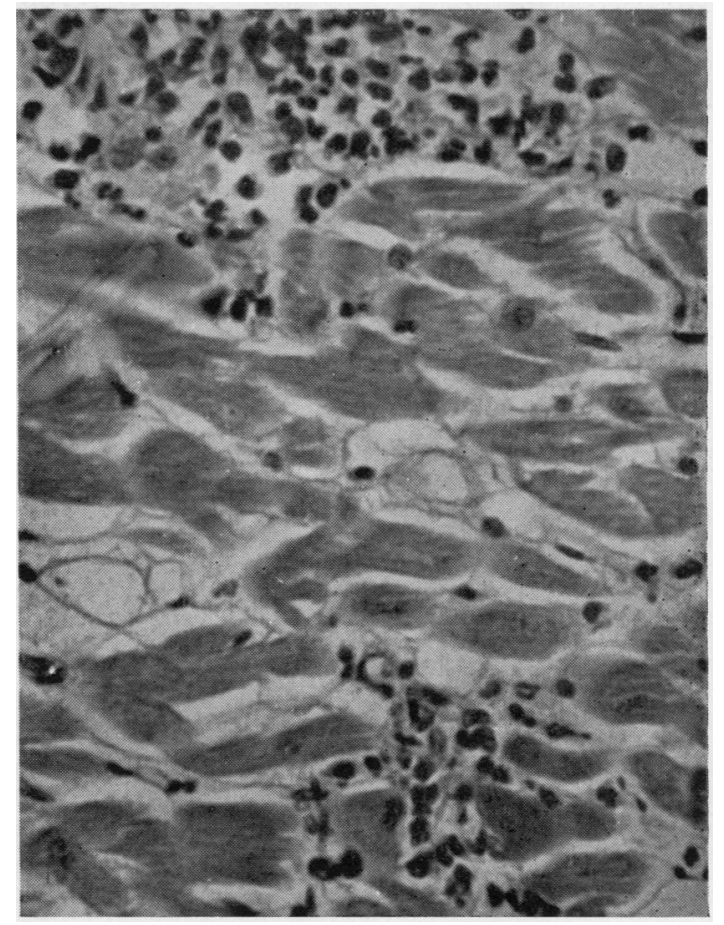

FIG. 6. High-power view of left atrium showing the cellular infiltrate. The cells were predominantly eosinophils and polymorphs. Haematoxylin and eosin, $\times 50$. 
and death. In the cases reported by Rusby and Wilson (1960) the longest period from haemoptysis to abnormal urine was eight months. However, case 2 of Scheer and Grossman (1964) had haemoptysis, dyspnoea, anaemia, and bilateral pulmonary infiltrates seven years before a renal lesion appeared.

Polyarteritis nodosa was considered because of the multiple disorders. Wood (1956) considered that polyarteritis should be suspected if an obscure cardiopathy is accompanied by other systemic diseases. Sampson (1945) stated that polyarteritis may affect the anterior segment of the eyes only. The bilateral penetrating corneal ulcers in this patient were a cause of great concern. Their healing when prednisone was given was quite unexpected. Schmidt (1964) described a 12-yearold boy with a history of haemoptysis for $10 \frac{1}{2}$ years, radiographic changes of fine diffuse pulmonary infiltrate thought to be primary pulmonary haemosiderosis with necropsy confirmation of this, plus evidence of polyarteritis nodosa in the lungs and kidneys. Rose and Spencer (1957) did not include the appearances of pulmonary haemosiderosis in their account of pulmonary polyarteritis nodosa. The mild peripheral neuropathy.could have been explained by the diabetes. The lymphadenopathy was not specific.

Although the patient had concurrent diabetes mellitus, there was no suggestion of haemochromatosis. Neither haemochromatosis nor posttransfusion haemosiderosis produce significant iron deposits in the lungs (Lancet, 1958). Court (1962) described a boy aged 12 years with diabetes mellitus and idiopathic pulmonary haemosiderosis. His patient's symptoms of cough and anaemia appeared to be controlled by prednisone in a dose of $15 \mathrm{mg}$. per day, but not by a smaller dose. The prednisone did not appear to influence the diabetic state, and insulin requirements were recorded as falling from 88 units per day to 72 units per day.

Massive doses of steroids are stated to be the only treatment of undisputed usefulness for exacerbations of idiopathic pulmonary haemosiderosis (Lancet, 1963). Soergel and Sommers (1962) considered that the short-term use of steroids helped during the bleeding episodes, but that longterm steroid therapy did not alter the course or prognosis of the disease. Our patient had neither anaemia nor haemoptysis at the time that steroids were given and there was no evidence that the prednisone altered his lung lesion in any way. It was hoped that the Stokes-Adams syncope would be assisted by steroid therapy. It had been temporarily improved when oral ephedrine was first given, and similarly it improved temporarily when prednisone was first given. When this effect? later appeared to have lessened, the dose of $\overline{\underline{\underline{0}}}$. prednisone was reduced. Perhaps it should have been increased: Pay and Waverley (1961) $\mathbb{Q}$ considered that for the treatment of intermittentes heart block the dosage of prednisolone should be$80 \mathrm{mg}$. per day. Alternatively, corticotrophin might have been worth a trial: Litchfield, Manley, and $\vec{\omega}$ Polak (1958) found in one of their cases that corticotrophin, 120 units per day, was effective where prednisone, $60 \mathrm{mg}$. per day, and cortisone, 0 $200 \mathrm{mg}$. per day, had failed.

Although the patient had streptococci in thei throat swab and a raised anti-streptolysin titre,,. there was no evidence that the carditis was? rheumatic.

\section{SUMMARY}

A patient is described with apparent idiopathico pulmonary haemosiderosis first detected on a chesto radiograph at the age of 22 years. Haemoptysis and iron deficiency anaemia appeared two years later. Irregular heart action appeared at the agea of 24 and was responsible for death from StokesAdams syncope at the age of 25 . The pulmonary haemosiderosis. was diffuse and was associatedo을 with marked thickening of the alveolar walls. The heart showed an active myocarditis with consider able areas of scarring. There was a cellular infiltrate of neutrophils and of eosinophils at the time of death.

In addition the patient had diabetes mellitus. found first at the age of 19 years. He had a mild 3 peripheral neuropathy and he had lymphadeno-i pathy with germinal centre hyperplasia.

Penetrating corneal ulcers affecting both eyes had been troublesome since the age of 19 years. When 25 years old both eyes were losing aqueous, $\frac{D}{0}$ but this leakage ceased when prednisone was given. The eyes showed a mild diffuse uveitis, but ${ }_{\sigma}^{N}$ the penetrating corneal ulcers were not adequately explained. There was no evidence of polyarteritis nodosa in the lungs or in any other tissues.

The records and radiographs of the Townsvilleco Hospital were made available by Dr. N. Scott-Young, the Medical Superintendent. In Townsville the patient $\stackrel{\oplus}{+}$ was under the care of Dr. R. A. Douglas. He was $\square$ first referred to Brisbane under Professor J. Tyrer, $\bar{O}$ and was transferred to the Ophthalmic Ward of the $\overrightarrow{\mathbb{D}}$ Princess Alexandra Hospital under Dr. O. Salkeld.

Dr. J. Little was responsible for the biopsy reports $\varrho$ and for supervision of the necropsy. Thoracotomy was performed by Dr. C. Lomas. Dr. A. Burry and $?$ Mr. D. Crowley provided the photographs. 


\section{REFERENCES}

Cameron, A. H. (1956). Pulmonary haemosiderosis associated with bronchiectasis. Thorax, 11, 105.

Campbell, S., and Macafee, C. A. J. (1959). A case of idiopathic pulmonary haemosiderosis with myocarditis. Arch. Dis. Childh., 34,218

Court, J. M. (1962). Idiopathic pulmonary haemosiderosis. Med. J. Aust., 1, 856.

Karlish, A. J. (1962). Idiopathic pulmonary haemosiderosis with unusual features. Proc, roy. Soc. Med., 55, 223.

Lancet (1958). Annotation: Idiopathic pulmonary haemosiderosis, 1 , 520

(1963). Leading article: Idiopathic pulmonary haemosiderosis, $1,979$.

Lendrum, A. C., Scott, L. D. W., and Park, S. D. S. (1950). Pulmonary changes due to cardiac disease with special reference to haemosiderosis. Ouart.J. Med., 19, 249.

Litchfield, J. W., Manley, K. A., and Polak, A. (1958). Stokes-Adams attacks treated with corticotrophin. Lancet, 1,935.

Pay, B. W., and Waverley, the Viscount (196i). Adrenocortical steroids in intermittent heart-block. Brit. med. J., 2, 139

Reye, D. (1945). Pulmonary haemosiderosis. Med. J. Aust., i, 35.
Rose, G. A., and Spencer, H. (1957). Polyarteritis nodosa. Quart. J. Med., 26, 43.

Rusby, N. L., and Wilson, C. (1960). Lung purpura with nephritis. Ibid., 29, 501

Sampson, R. (1945). Periarteritis nodosa affecting the eye. Brit. $J$. Ophthal. , 29, 282.

Scheer, R. L., and Grossman, M. A. (1964). Immune aspects of the glomerulonephritis associated with pulmonary hemorrhage. Ann. intern. Med., 60, 1009.

Schmidt, H. W. (1964). Lung purpura and pulmonary hemosiderosis. Med. Clin. N. smer., 48, 1011.

Soergel, K. H., and Sommers, S. C. (1962). Idiopathic pulmonary hemosiderosis and related syndromes. Amer. J. Med., 32, 499.

Walton, M., and Williams, A. A. (1951). Idiopathic pulmonary haemosiderosis. Brit. med. J., 2, 390

Wood, P. (1954). An appreciation of mitral stenosis. Part II. Investigations and results. Ibid., 1, 1113 . (1956). Diseases of the Heart and Circulation, 2nd ed., p. 624 Eyre and Spottiswoode, London.

Wyllie, W. G., Sheldon, W., Bodian, M., and Barlow, A. (1948). Idiopathic pulmonary haemosiderosis (Essential brown induration of the lungs). Ouart. J. Med., 17, 25 .

Wynn-Williams, N., and Young, R. D. (1956). Idiopathic pulmonary haemosiderosis in an adult. Thorax, 11, 101. 